\section{Cinderella goes to the ball}

\author{
After decades of \\ neglect, research into \\ 'innate' immunity has \\ moved to centre stage. \\ Phyllida Brown explores \\ the excitement that \\ now surrounds an \\ evolutionarily ancient \\ arm of our immune \\ system.
}

$\mathrm{T}$ he innate immune response - an inflammatory reaction that helps control infections in their early stages - has long been the Cinderella of immunology. For decades, innate immunity was dismissed as an evolutionary throwback, providing no more than a quick-anddirty holding operation before giving way to the exquisitely complex adaptive immune response. Studies into how the adaptive immune system churns out cells and antibodies tailored to recognize particular pathogens attracted all the glamour.

But now all that is changing. Over the past few years, researchers have begun to realize that the innate immune response is a powerful screening tool. In the early hours of an infection, it can distinguish between different classes of pathogenic bacteria, viruses and fungi. And it is becoming clear that the innate immune response is also crucial for prodding the slower-acting adaptive system into action. "The field has suddenly heated up," says Alan Aderem, an immunologist at the Institute for Systems Biology in Seattle.

It is innate immunity that causes the redness and swelling that soon erupts around an infected wound, and the initial aches and fever that precede a bout of flu. Such reactions help to prevent invading pathogens from spreading rapidly around the body — which is important, because the adaptive immune system might not kick in for several days.

The chief cause of the fresh excitement is a rapidly accumulating body of research into a family of molecules called Toll-like receptors

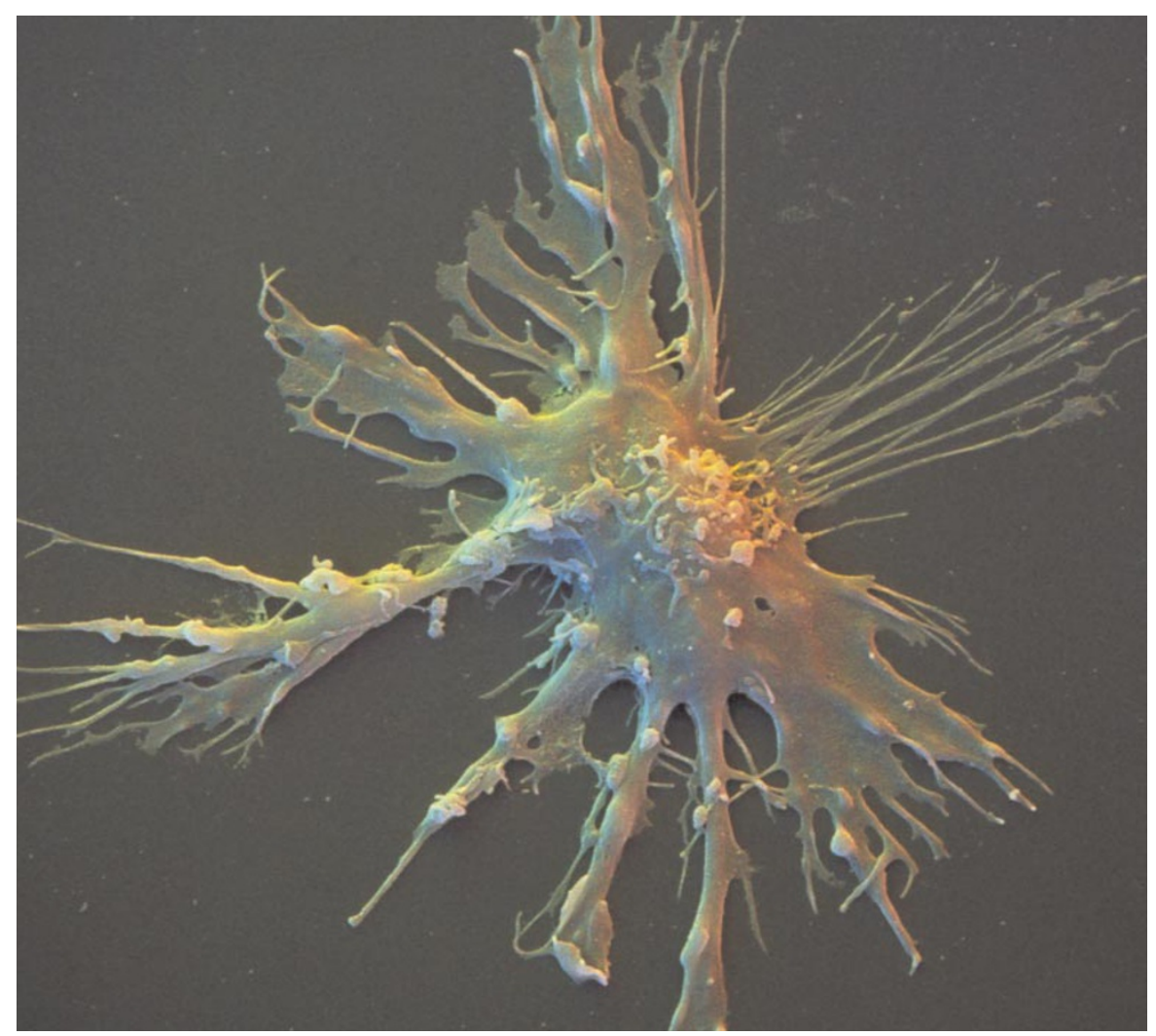

Trigger happy: receptors on dendritic cells help to fire up the innate immune response.

(TLRs), which have a central role in triggering innate immune responses. These receptors sit on the surface of two classes of mammalian white blood cells macrophages and dendritic cells — and on the cells of certain other tissues such as the epithelia that line our guts and nasal passages.

The TLRs owe their name to a closely related receptor called Toll, first identified in the fruitfly Drosophila. Invertebrates lack an adaptive immune system, but when Toll and its allies are stimulated by the presence of foreign microorganisms, they initiate the production of antimicrobial compounds that target the invaders. Mammalian innate immunity works differently, producing molecules called cytokines that cause inflammation. But the fact that the TLRs are so similar to Toll reveals that they are part of an ancient defence mechanism that has been conserved through evolution.

\section{Screen test}

Researchers are now discovering that each type of TLR recognizes specific molecular patterns carried by different pathogens. These pathogen-associated molecular patterns, or PAMPs, are usually embedded in structures that are essential to their bearers' survival. As such, they evolve slowly and tend to be shared by whole classes of bacteria, viruses or fungi. And, as different classes of microbe carry different PAMPs, a macrophage or dendritic cell can use its family of TLRs to classify the invader, and respond appropriately. "What we are starting to see is a digital information system, rather like barcode recognition," says Aderem.

Since the first mammalian TLR, now called TLR4, was identified in 1997 by Charles Janeway and Ruslan Medzhitov at Yale University in New Haven, Connecticut ${ }^{1}$, nine more have been added to the list ${ }^{2-5}$. Researchers have also made rapid progress in linking individual TLRs with particular PAMPs. TLR4 is activated by lipopolysaccharide (LPS), a component of the cell wall of Gram-negative bacteria such as Salmonella ${ }^{6}$. It has since been shown also to recognize other bacterial PAMPs, and an important viral protein ${ }^{8}$. TLR2, meanwhile, responds to a glycolipid called lipoarabinomannan ${ }^{9,10}$, a component of the cell walls of mycobacteria, a group that includes the tuberculosis pathogen. It is also activated by the LPS of Leptospira bacteria ${ }^{11}$. And in this issue of Nature ${ }^{12}$, Aderem's team shows that TLR5 is activated by flagellin, a protein found on the surface of flagellate bacteria such as Listeria.

With some TLRs responding to more than one PAMP, and other TLRs operating together, recognizing combinations of PAMPs specific to particular classes of pathogen ${ }^{13}$, Medzhitov argues that the 10 known receptors can deal with around 20 specific PAMP combinations. "That's enough to recognize practically any class of infective agent," he says.

Once activated by a PAMP, a TLR triggers a cascade of cellular signals. Most researchers 

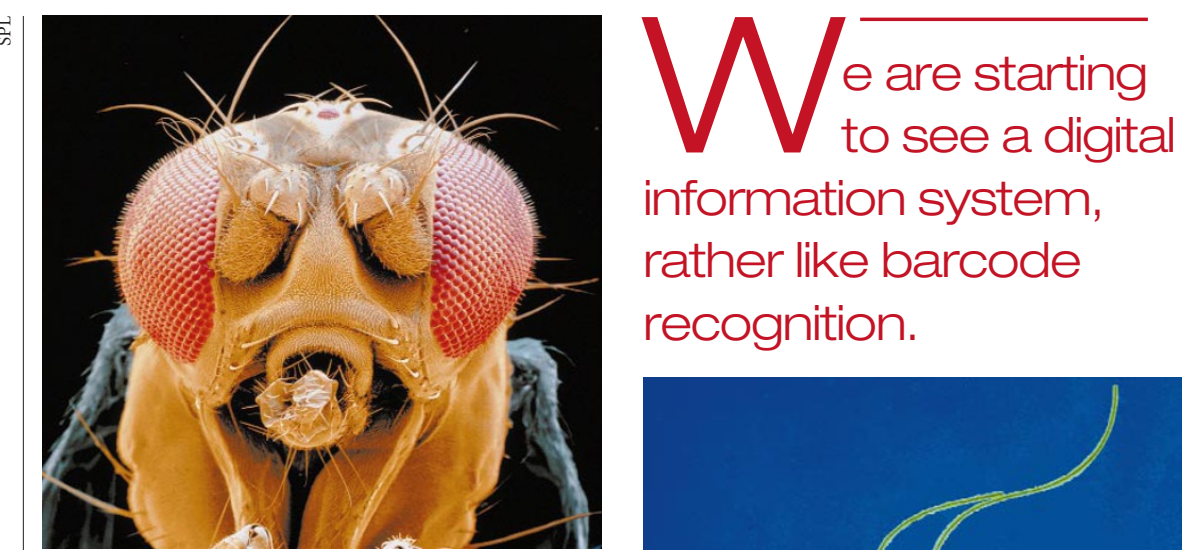
information system, rather like barcode recognition.
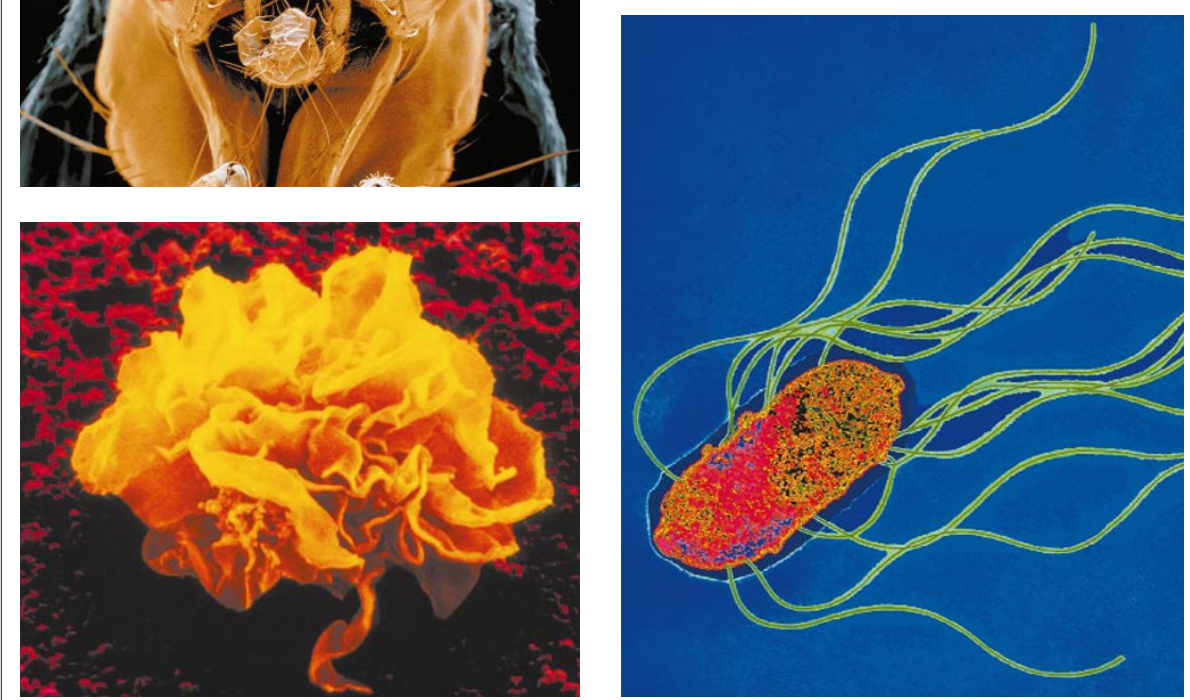

On the defensive: the 'Toll-like' receptors on cells such as macrophages (bottom left) can recognize invading pathogens such as Salmonella (right) and kick-start an immune response. They derive their unusual name from similar proteins found in fruitflies (top).

have so far focused on signalling pathways that eventually activate a transcription factor called NF- $\kappa B$, which regulates the activity of the genes that produce cytokines such as tumour necrosis factor- $\alpha$ and interleukin-1. But there is evidence that other signalling pathways are also switched on when TLRs are activated ${ }^{14}$. In general, the cytokines make blood vessels leakier at the site of infection, allowing fluid and proteins to pass into the tissues. Cytokines also attract other white blood cells to the scene.

\section{Concerted effort}

Exactly how PAMPs activate their corresponding TLRs remains largely mysterious, and it seems likely that other 'helper' molecules are involved. For example, on the surface of Gram-negative bacteria, LPS is complexed with another molecule called LPS-binding protein (LBP). This complex is recognized by a molecule called CD14, which is carried by macrophages ${ }^{15}$. Immunologists believe that it is probably the trio of LPS, LBP and CD14 that activates TLR4 (ref. 14).

Although hard evidence is still scant, most researchers suspect that different TLRs trigger subtly different combinations of cytokines, each appropriate for different classes of infectious agent. For example, one might expect that TLRs that recognize viral proteins would trigger the release of cytokines that instruct other cells to make potent antiviral comping roles of different signalling pathways and cytokines make it difficult to turn this speculation into proven fact.

The buzz surrounding TLRs is not just a result of their role in triggering innate immune responses, however. There is also growing evidence that activated TLRs send vital wake-up signals to the adaptive immune system. "Until TLRs were discovered, the innate immune system was considered to be just an evolutionary rudiment," says Medzhitov. "But we now know that it

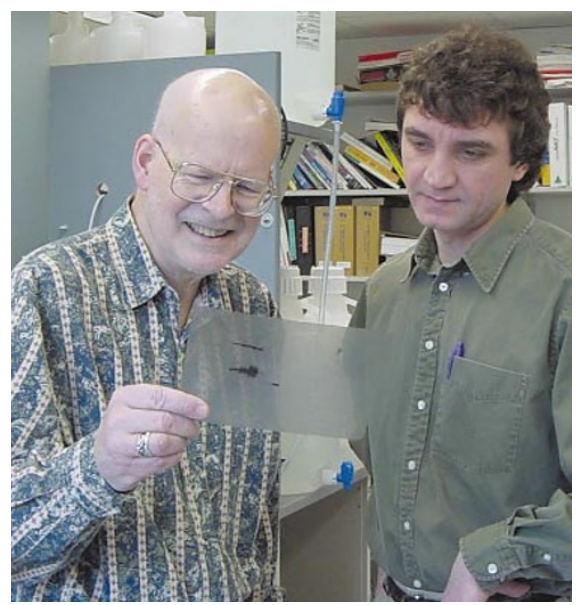

Receptive audience: Charles Janeway (left) and Ruslan Medzhitov were the first to identify Tolllike receptors in mammalian cells. pounds such as interferons. But the overlap- plays a key role in adaptive immunity."

Janeway has long championed this idea. Back in 1989, when he first suggested that the innate immune response is triggered by microbial molecules such as LPS, he also argued that the same signals trigger the innate system to activate 'naïve' cells of the adaptive system and turn them into armed and proliferating attackers ${ }^{16}$. Janeway based his argument on studies of adjuvants, components that must be added to most vaccines to ensure that they provoke a sufficiently vigorous immune response. Many adjuvants contain bacterial molecules, and Janeway argued that these are recognized by the innate immune system, which prods the adaptive system into action.

$\mathrm{T}$ cells are key players in the adaptive immune system. Different T cells carry different receptors that recognize distinctive molecules known as antigens. At any one time, the immune system includes a vast array of circulating $\mathrm{T}$ cells with different specificities, each present in small numbers. When the body comes under attack, the relevant $\mathrm{T}$ cells are activated and proliferate rapidly. (Similar processes underlie the production of specific antibodies by B cells.)

\section{For whom the cell tolls}

The key to effective adaptive immunity lies in ensuring that this activation only occurs in response to antigens that present a threat - reactions to most of the body's own proteins, or innocuous antigens such as those in our food, should be avoided. Immunologists have long known that macrophages and dendritic cells are involved in processing antigens so that they can be recognized by $\mathrm{T}$ cells. But these antigen-presenting cells only activate $\mathrm{T}$ cells when they also produce 'co-stimulatory' molecules.

The fact that macrophages and dendritic cells are also involved in innate immunity might have suggested a link between the two arms of the immune system. But supporting evidence for Janeway's hypothesis that innate immune responses help trigger adaptive immunity has been boosted by studies of the TLRs. The best known of the co-stimulatory molecules belong to a group of proteins called B7, produced by macrophages and dendritic cells. When Janeway and his colleagues discovered TLR4 in 1997, they showed that its activation causes the cells involved to produce $\mathrm{B} 7$ in addition to cytokines ${ }^{1}$.

Then, last December, researchers led by Shizuo Akira of Osaka University in Japan added another line of evidence. They observed that sequences found mainly in bacterial DNA and rarely in the DNA of higher organisms - called CpG repeats are recognized by TLR9 (ref. 17). As CpG repeats in vaccines that are based on naked DNA stimulate the adaptive immune system to respond to these vaccines, this strength-

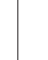


ened the link between the two arms of the immune system.

The latest twist in the tale is that the TLRs might also help to explain a problem that has long puzzled immunologists: why innate and adaptive immune responses are sometimes triggered by the body's own proteins in the absence of any infection. For example, when tissue is damaged in major surgery, the area around the incision sees soaring levels of cytokines and inflammation, however sterile the conditions. This inflammatory response can also trigger a transient burst of antibodies against the body's own tissues.

\section{Danger signs}

In 1994, Polly Matzinger of the National Institute of Allergy and Infectious Diseases in Bethesda, Maryland, came up with a provocative suggestion ${ }^{18}$. She said that it was simplistic to argue that the immune system distinguishes between 'self' and 'foreign' antigens. Instead, Matzinger proposed that the key to triggering immunity is an association between an antigen and a 'danger' signal. In addition to molecules produced by pathogens, she said that the signals could include products of damaged tissue, such as proteins that only emerge from cells when their membranes are ruptured. In cellular terms, this is like entering a building and seeing blood spattered on the walls.

Evidence is now emerging that some TLRs may indeed be involved in responding to such danger signals. For example, last month, researchers at the University of Pennsylvania Medical Center in Philadelphia reported that fibronectin, a molecule released by damaged tissue, can activate TLR4 (ref. 19). This same receptor is activated by heat-shock proteins, which are produced by cells under stress ${ }^{20}$. Matzinger is delighted: "This is exactly what I have been saying."

As our understanding of the TLRs deepens, immunologists are starting to look for

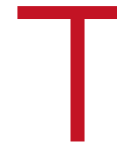
he innate immune response will never again be seen as a crude leftover from our evolutionary past.

variation in the genes encoding TLRs and their expression to see whether this affects our susceptibility to disease. "We have known for a long time that different individuals have different responses to bacteria, for example, and this could be one of the reasons," says Marco Colonna of the Basel Institute for Immunology in Switzerland. Some people, for instance, are particularly prone to Staphylococcus infections, and Aderem suggests that deficiencies in the production of certain TLRs, or the signalling pathways that lie downstream of the receptors, might explain why. If so, he says, it might be possible to devise therapies to circumvent the blocked signalling pathways.

Equally important, says Aderem, the receptors might also be involved in inflammatory diseases such as atherosclerosis, which is now widely believed to involve chronically activated macrophages. If certain TLR-related signalling pathways could be blocked without compromising vulnerability to infection, it might be possible, he argues, to design drugs that prevent the inflammatory process from running awry.

\section{A rousing chorus}

But there is one caveat. The TLRs are not the only recognition tool in the armoury of the innate immune system, nor are they its only way of waking up the adaptive immune system. Mammalian immune defences are famously rich in cells and cytokines with overlapping functions, and back-up signalling pathways. And evidence is already beginning to emerge for such secondary mechanisms that operate alongside the TLRs. In this issue of Nature ${ }^{21}$, Colonna and his colleagues report on experiments using mice in which they examined a different receptor, called TREM-1, which is carried by other types of white blood cells such as neutrophils.

TREM- 1 is activated by exposure to bacteria such as Pseudomonas aeruginosa or Staphylococcus aureus, although the precise molecular pattern it responds to is unknown. Colonna and his colleagues have now found that the receptor can start a signalling cascade that results in inflammation through a pathway that does not involve NF$\kappa B$. They have also found that TREM-1 may play a key role in septic shock, a systemic reaction that develops if massive quantities of inflammatory cytokines are released in

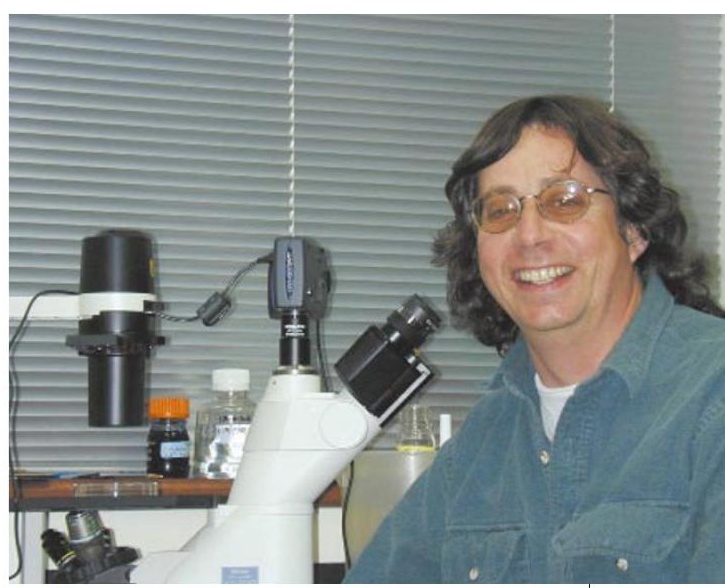

Cool it: Alan Aderem thinks that studies of Toll-like receptors will help to design treatments for inflammatory disease.

response to a heavy load of microorganisms, for example in peritonitis.

More evidence for the diversity of signalling pathways involved in innate immunity has come from Akira's group. Until recently, a protein called MyD88 was thought to be key to TLR-triggered signalling. But new research from Akira's team indicates that dendritic cells from mice lacking MyD88 can still activate T cells, suggesting that this aspect of TLR4-triggered signalling uses an alternative pathway ${ }^{22}$.

Clearly, there are plenty of unanswered questions. But as researchers scramble to uncover the workings of the TLRs and innate immunity, they may hit upon new strategies for the development of drugs and vaccines. One thing is for sure: the innate immune response will never again be regarded as a crude leftover from our evolutionary past. For Cinderella, the ball is far from over.

\section{Phyllida Brown is a science writer based in Exeter.}

1. Medzhitov, R., Preston-Hurlburt, P. \& Janeway, C. A. Jr Nature 388, 394-397 (1997).

2. Rock, F. L., Hardiman, G., Timans, J. C., Kastelein, R.A. \& Bazan, J. F. Proc. Natl Acad. Sci. USA 95, 588-593 (1998).

3. Chaudhary, P. M. et al. Blood 91, 4020-4027 (1998).

4. Takeuchi, O. et al. Gene 231, 59-65 (1999).

5. Chuang, T. \& Ulevitch, R. J. Biochim. Biophys. Acta 1518, 157-161 (2001).

6. Poltorak, A. et al. Science 282, 2085-2088 (1998).

7. Lien, E. et al. J. Biol. Chem. 274, 33419-33425 (1999).

8. Kurt-Jones, E. A. et al. Nature Immunol. 1, 398-401 (2000).

9. Means, T. K. et al. J. Immunol. 163, 3920-3927 (1999).

10. Underhill, D. M., Ozhinsky, A., Smith, K. D. \& Aderem, A. Proc. Natl Acad. Sci. USA 96, 14459-14463 (1999).

11. Werts, C. et al. Nature Immunol. 2, 346-352 (2001).

12. Hayashi, F. et al. Nature 410, 1099-1103 (2001).

13. Ozinsky, A. et al. Proc. Natl Acad. Sci. USA 97, 13766-13771 (2000).

14. Aderem, A. \& Ulevitch, R. J. Nature 406, 782-787 (2000).

15. Tobias, P. S., Soldau, K., Gegner, J. A., Mintz, D. \& Ulevitch, R. J. J. Biol. Chem. 270, 10482-10488 (1995).

16. Janeway, C. A. Jr Cold Spring Harbor Symp. Quant. Biol. 54, $1-13(1989)$.

17. Hemmi, H. et al. Nature 408, 740-745 (2000).

18. Matzinger, P. Annu. Rev. Immunol. 12, 991-1045 (1994). 19. Okamura, Y. et al. J. Biol. Chem. 276, 10229-10233 (2001). 20. Ohashi, K., Burkart V., Flohe, S. \& Kolb, H. J. Immunol. 164, 2064-2069 (2000)

21. Bouchon, A., Facchetti, F., Weigand, M. A. \& Colonna, M. Nature 410, 1103-1107 (2001).

22. Kaisho, T., Takeuchi, O., Kawai, T., Katsuaki, H. \& Akira, S. J. Immunol. 166, 5688-5694 (2001). 